\title{
LOCALLY MODULAR LATTICES AND LOCALLY DISTRIBUTIVE LATTICES
}

\author{
SHÛICHIRÔ MAEDA
}

\begin{abstract}
A locally modular (resp. locally distributive) lattice is a lattice with a congruence relation and each of whose equivalence class has sufficiently many elements and is a modular (resp. distributive) sublattice. Both the lattice of all closed subspaces of a locally convex space and the lattice of projections of a locally finite von Neumann algebra are locally modular. The lattice of all $T_{1}$-topologies of an infinite set is locally distributive.
\end{abstract}

Introduction. In this paper, a lattice $L$ is called locally modular (resp. locally distributive) when $L$ has a congruence relation $\theta$ such that each equivalence class by $\theta$ which contains sufficiently many elements is a modular (resp. distributive) sublattice. Any locally distributive lattice is locally modular evidently, and it is shown in $\$ 1$ that any locally modular lattice is both upper and lower semimodular in the sense of Birkhoff [2]. Moreover in this section it is proved that both the lattice of all closed subspaces of a locally convex space and the lattice of all projections of a locally finite von Neumann algebra are locally modular.

It was proved by Larson and Thron [5] that the lattice of all $T_{1}$-topologies on an infinite set is both upper and lower semimodular. Generalizing this result, it is shown in $\$ 2$ that the lattice of all $T_{1}$-topologies is locally distributive. Moreover, the final theorem of [5] is formulated as a theorem on locally distributive lattices.

In the last section, we determine the form of standard elements in the dual of the lattice of $T_{1}$-topologies. This result shows us that this lattice has infinitely many standard elements but has no neutral elements except 0 and 1.

1. Locally modular lattices. An equivalence relation $\theta$ in a lattice $L$ is called a congruence relation when it satisfies the following condition:

$$
\begin{aligned}
& \text { If } a_{1} \equiv b_{1}(\theta) \text { and } a_{2} \equiv b_{2}(\theta) \\
& \text { then } a_{1} \vee a_{2} \equiv b_{1} \vee b_{2}(\theta) \text { and } a_{1} \wedge a_{2} \equiv b_{1} \wedge b_{2}(\theta) \text {. }
\end{aligned}
$$

Received by the editors May 8, 1973.

AMS (MOS) subject classifications (1970). Primary 06A30, 06A35; Secondary 46A05, 46L10, 54A10, 54D10.

Key words and phrases. Locally modular lattice, locally distributive lattice, semimodular, lattice of $T_{1}$-topologies, standard element.

(c) American Mathematical Society 1974 
Then, for any $a \in L$, the equivalence class $[a]=\{x \in L ; x \equiv a(\theta)\}$ is a sublattice of $L$. Moreover, if $x, y \in[a]$ and $x<y$ then the interval $L[x, y]=\{z \in L ; x \leqq z \leqq y\}$ is contained in [a] (see [2, p. 27]).

In a lattice, we write $a<\cdot b$ when $b$ covers $a$.

Definition. A lattice $L$ is called locally modular when there exists a congruence relation $\theta$ in $L$ satisfying the following three conditions:

$\left(\theta_{1}\right)$ If $a \neq 1$ in $L$ then there exists $b \in L$ such that $b>a$ and $b \equiv a(\theta)$, and if $a \neq 0$ then there exists $b \in L$ such that $b<a$ and $b \equiv a(\theta)$.

$\left(\theta_{2}\right)$ If $a<\cdot b$ then $a \equiv b(\theta)$.

$\left(\theta_{M}\right)$ For any $a \in L$, the sublattice $[a]$ is modular.

$L$ is called locally distributive when, in the above definition, $\left(\theta_{M}\right)$ is replaced by the following condition:

$\left(\theta_{D}\right)$ For any $a \in L$, the sublattice $[a]$ is distributive.

Evidently, any locally distributive lattice is locally modular. The two conditions $\left(\theta_{1}\right)$ and $\left(\theta_{2}\right)$ assert that each sublattice [a] contains sufficiently many elements.

THEOREM 1.1. Any locally modular lattice $L$ is both upper and lower semimodular in the sense of Birkhoff [2].

PRoof. Let $a \wedge b<\cdot a$ and $a \wedge b<\cdot b$ in $L$. Then we have $a \equiv b(\theta)$ by $\left(\theta_{2}\right)$, and hence $(a, b) M^{*}$ and $(b, a) M^{*}$ by $\left(\theta_{M}\right)$ (see (1.7) of [6]). Hence we have $b<\cdot a \vee b$ and $a<\cdot a \vee b$ by (7.5.4) of [6]. Thus $L$ is upper semimodular. Similarly we can prove that $L$ is lower semimodular.

A lattice $L$ with 0 and 1 is called a $D A C$-lattice when both $L$ and its dual $L^{*}$ are atomistic lattices with the covering property (see [6, §27]). We shall prove that any DAC-lattice is locally modular. We write $\mathscr{F}(L)$ for the set of all finite elements and write $\Omega(L)$ for the set of all atoms of $L$.

LEMmA 1.1. Let $a$ and $b$ be elements of a DAC-lattice $L$.

(i) There exists $u \in \mathscr{F}(L)$ such that $a \vee u=b$ if and only if there exists $u^{*} \in \mathscr{F}\left(L^{*}\right)$ such that $b \wedge u^{*}=a$.

(ii) There exists $u \in \mathscr{F}(L)$ such that $a \vee u=b \vee u$ if and only if there exists $u^{*} \in \mathscr{F}\left(L^{*}\right)$ such that $a \wedge u^{*}=b \wedge u^{*}$.

Proof. (i) If $a \vee u=b$ with $u \in \mathscr{F}(L)$, then by the covering property there exists a connected chain $a=x_{0}<\cdot x_{1}<\cdots<\cdot x_{n}=b$. Since $L$ is dual-atomistic, there exist dual atoms $h_{i}(i=1, \cdots, n)$ such that $h_{i} \geqq x_{i-1}$ and $h_{i} \geq x_{i}$. Putting $u^{*}=h_{1} \wedge \cdots \wedge h_{n}$, we have $u^{*} \in \mathscr{F}\left(L^{*}\right)$ and $b \wedge u^{*}=a$. The converse statement can be proved similarly. Moreover, it is easily seen that the statement (ii) follows from (i).

THEOREM 1.2. Let L be a DAC-lattice. $L$ is locally modular if we define $a \equiv b(\theta)$ by $a \vee u=b \vee u$ for some $u \in \mathscr{F}(L)$. 
Proof. It is evident that $\theta$ is an equivalence relation. $\theta$ is a congruence relation by Lemma 1.1 (ii), and it satisfies $\left(\theta_{1}\right)$ since $L$ is atomistic and dual-atomistic. It satisfies $\left(\theta_{2}\right)$ evidently. When $a \equiv b(\theta)$, there exists $u^{*} \in \mathscr{F}\left(L^{*}\right)$ with $a \wedge u^{*}=b \wedge u^{*}=c$. It follows from Lemma 1.1 that there exist $u, v \in \mathscr{F}(L)$ such that $c \vee u=a, c \vee v=b$. Since $L$ is finite-modular by $(27.6)$ of [6], we have $(a, b) M^{*}$ by $(27.12)$ of [6]. Hence, $\theta$ satisfies $\left(\theta_{M}\right)$.

By (31.10) of [6], this theorem implies the following result.

COROLlary. The lattice of all closed subspaces of a locally convex space is locally modular.

Next, let $L$ be a relatively complemented lattice with 0 and 1 . The following condition is considered in $\$ 35$ of [6]:

(J) $L$ has a join-dense $p$-ideal $J$ whose elements are all modular.

It follows from (35.6) of [6] that $L^{*}$ also satisfies (J) by using $J^{*}=$ $\left\{x \in L ; x\right.$ has a complement $\left.x^{\prime} \in J\right\}$ instead of $J$. An important example of such a lattice is a locally finite dimension lattice defined in (35.15) of [6].

LEMMA 1.2. Let $a$ and $b$ be elements of $a$ relatively complemented lattice $L$, with 0 and 1 , satisfying $(\mathrm{J})$.

(i) There exists $u \in J$ such that $a \vee u=b$ if and only if there exists $u^{*} \in J^{*}$ such that $b \wedge u^{*}=a$.

(ii) There exists $u \in J$ such that $a \vee u=b \vee u$ if and only if there exists $u^{*} \in J^{*}$ such that $a \wedge u^{*}=b \wedge u^{*}$.

Proof. If $a \vee u=b$ with $u \in J$, then taking a complement $u^{*}$ of $b$ in the interval $L[a, 1]$, we have $b \wedge u^{*}=a$ and $u \vee u^{*}=u \vee a \vee u^{*}=b \vee u^{*}=1$. Hence, $u^{*} \in J^{*}$ by the statement (1) in the proof of (35.6) of [6]. The converse statement can be proved similarly. The statement (ii) follows from (i).

THEOREM 1.3. Let $L$ be a relatively complemented lattice, with 0 and 1 , satisfying (J). $L$ is locally modular if we define $a \equiv b(\theta)$ by $a \vee u=b \vee u$ for some $u \in J$.

Proof. It follows from Lemma 1.2 that $\theta$ is a congruence relation. $\theta$ satisfies $\left(\theta_{1}\right)$ since $J$ (resp. $J^{*}$ ) is join-dense in $L$ (resp. $L^{*}$ ). It satisfies $\left(\theta_{2}\right)$ evidently. When $a \equiv b(\theta)$, we can prove $(a, b) M^{*}$ by the same way as in the proof of Theorem 1.2, using (35.10) of [6] instead of (27.12).

Corollary. Any locally finite dimension lattice is locally modular. Especially, the lattice of all projections of a locally finite $A W^{*}$-algebra is locally modular (see (37.16) of [6]). 


\section{Locally distributive lattices.}

LemMA 2.1. Let $L$ be an atomistic lattice. A congruence relation $\theta$ in $L$ satisfies the condition $\left(\theta_{D}\right)$ if it satisfies the following condition:

$\left(\Omega_{D}\right)$ If $a \equiv b(\theta)$ in $L$ and if $p$ is an atom of $L$ such that $p \leqq a \vee b$ then either $p \leqq a$ or $p \leqq b$.

Proof. For $x, y, z \in[a]$, we have $(x \vee y) \wedge z=(x \wedge z) \vee(y \wedge z)$, since if $p$ is an atom with $p \leqq(x \vee y) \wedge z$ then $p \leqq(x \wedge z) \vee(y \wedge z)$ by $\left(\Omega_{D}\right)$. Similarly, $(x \wedge y) \vee z=(x \vee z) \wedge(y \vee z)$ holds.

Lemma 2.2. Let $L$ be a locally distributive atomistic lattice whose congruence relation $\theta$ satisfies $\left(\Omega_{D}\right)$. If $x<\cdot a, y<\cdot a$ in $L$ and if there exists an atom $p$ of $L$ such that $a=x \vee p=y \vee p$ then $x=y$.

Proof. Evidently $p$ and $p$. Since $x \equiv a \equiv y(\theta)$ by $\left(\theta_{2}\right)$, we have $p \leq x \vee y$ by $\left(\Omega_{D}\right)$, and hence $x \vee y<a$. Since $x<\cdot a$ we have $x=x \vee y$, and similarly $y=x \vee y$.

THEOREM 2.1. Let $L$ be a complete locally distributive atomistic lattice whose congruence relation $\theta$ satisfies $\left(\Omega_{D}\right)$. For any $a \in L$, we put $\Gamma(a)=\{x \in L ; x<\cdot a\}$. If we put $a(M)=\bigwedge(x ; x \in M)$ for every subset $M$ of $\Gamma(a)(a(\varnothing)=a)$, then the set $\{a(M) ; M \subset \Gamma(a)\}$ is a complete sublattice of $L$ which is dual isomorphic to the Boolean lattice formed by all subsets of $\Gamma(a)$.

Proof. Let $\left\{M_{\alpha} ; \alpha \in I\right\}$ be an arbitrary family of subsets of $\Gamma(a)$. The equation $a\left(\bigcup_{\alpha} M_{\alpha}\right)=\bigwedge_{\alpha} a\left(M_{\alpha}\right)$ holds evidently and we shall prove $a\left(\bigcap_{\alpha} M_{\alpha}\right)=\bigvee_{\alpha} a\left(M_{\alpha}\right)$ (we denote by $\cup$ and $\cap$ the union and the intersection respectively). It suffices to show that if $p$ is an atom with $p \leqq a$ and $p \leq \bigvee_{\alpha} a\left(M_{\alpha}\right)$ then $p a\left(\bigcap_{\alpha} M_{\alpha}\right)$. For every $\alpha$, there exists $x_{\alpha} \in M_{\alpha}$ with $p$ 年 $x_{\alpha}$, since $p a\left(M_{\alpha}\right)$. Then, since $x_{\alpha} \vee p=a$, it follows from Lemma 2.2 that $x_{\alpha}=x_{\beta}$ for every $\alpha, \beta \in I$. Hence, $p \leq a\left(\bigcap_{\alpha} M_{\alpha}\right)$. Therefore, $\{a(M) ; M \subset$ $\Gamma(a)\}$ is a complete sublattice of $L$. Moreover, it is easy to prove by Lemma 2.2 that the mapping $M \rightarrow a(M)$ is one-to-one. This completes the proof.

Next, we shall give an example of a locally distributive lattice whose congruence relation satisfies $\left(\Omega_{D}\right)$. Let $X$ be an infinite set. A topology on $X$ is denoted by the collection $\mathscr{T}$ of all open sets. $\mathscr{T}$ is a $T_{1}$-topology if and only if $\mathscr{T}$ contains all cofinite subsets of $X$. The set $L_{T}(X)$ of all $T_{1}$-topologies on $X$ forms a complete lattice, ordered by set inclusion, that is, $\mathscr{T}_{1}<\mathscr{T}_{2}$ means that $\mathscr{T}_{2}$ is finer than $\mathscr{T}_{1}$. The greatest element of $L_{T}(X)$ is the discrete topology and the least element is the cofinite topology (see $[7, \S 1])$.

For any subset $Y$ of $X$, we denote by $\mathscr{P}(Y)$ the collection of all subsets of $Y$. It was shown in [3] and [7] that a dual-atom of $L_{T}(X)$, which is called 
a nonprincipal ultratopology, has the form

$$
\mathscr{T}(x, \mathscr{U})=\mathscr{P}(X-\{x\}) \cup \mathscr{U}
$$

where $x \in X$ and $\mathscr{U}$ is a nonprincipal ultrafilter on $X$, and it follows from Theorem 1.1 of [7] that $L_{T}(X)$ is dual-atomistic. We remark that $L_{T}(X)$ is not atomistic.

THeOREM 2.2. Let $X$ be an infinite set. The lattice $L_{T}(X)$ of $T_{1}$-topologies on $X$ is locally distributive if we define $\mathscr{T}_{1} \equiv \mathscr{T}_{2}(\theta)$ by $\mathscr{T}_{1} \cap \mathscr{P}(X-F)=$ $\mathscr{T}_{2} \cap \mathscr{P}(X-F)$ for some finite subset $F$ of $X$ (i.e. $\mathscr{T}_{1}$ coincides with $\mathscr{T}_{2}$ on some cofinite subset). Moreover, this congruence relation $\theta$ satisfies $\left(\Omega_{D}\right)$ in the dual of $L_{T}(X)$.

Proof. It is easy to verify that $\theta$ is a congruence relation. Let $\mathscr{T} \in L_{T}(X)$. If $\mathscr{T}$ is not discrete, then there exists $x \in X$ such that $\{x\} \notin \mathscr{T}$. Putting $\mathscr{T}_{1}=\mathscr{T} \cup\{G \cup\{x\} ; G \in \mathscr{T}\}$, we have $\mathscr{T}<\mathscr{T}_{1} \in L_{T}(X)$ and $\mathscr{T}_{1} \equiv \mathscr{T}(\theta)$. If $\mathscr{T}$ is not the cofinite topology, then there exists a dualatom $\mathscr{T}(x, \mathscr{U})$ such that $\mathscr{T}(x, \mathscr{U}) \geq \mathscr{T}$. Putting $\mathscr{T}_{2}=\mathscr{T} \wedge \mathscr{T}(x, \mathscr{U})$, we have $\mathscr{T}_{2}<\mathscr{T}$ and $\mathscr{T}_{2} \equiv \mathscr{T}(\theta)$. Hence, $\theta$ satisfies $\left(\theta_{1}\right)$. If $\mathscr{T}_{1}<\cdot \mathscr{T}_{2}$, then there exists a dual-atom $\mathscr{T}(x, \mathscr{U})$ such that $\mathscr{T}_{1}=\mathscr{T}_{2} \wedge \mathscr{T}(x, \mathscr{U})$. Hence, $\theta$ satisfies $\left(\theta_{2}\right)$.

Next, we shall show that $\theta$ satisfies $\left(\Omega_{D}\right)$ in the dual of $L_{T}(X)$, that is, if $\mathscr{T}_{1} \cap \mathscr{P}(X-F)=\mathscr{T}_{2} \cap \mathscr{P}(X-F)$ and $\mathscr{T}(x, \mathscr{U}) \geqq \mathscr{T}_{1} \wedge \mathscr{T}_{2}$ then $\mathscr{T}(x, \mathscr{U}) \geqq \mathscr{T}_{1}$ or $\mathscr{T}_{2}$. If we had $\mathscr{T}(x, \mathscr{U}) \geq \mathscr{T}_{i}$ for $i=1,2$, then there would exist $G_{i} \in \mathscr{T}_{i}$ such that $G_{i} \notin \mathscr{T}(x, \mathscr{U})$. Since $G_{i} \notin \mathscr{P}(X-\{x\}) \cup \mathscr{U}$, we have $x \in G_{i} \notin \mathscr{U}$, and then $G_{1} \cup G_{2} \notin \mathscr{U}$ since $\mathscr{U}$ is an ultrafilter. We put

$$
G=\left(G_{1} \cup G_{2}\right) \cap\left\{G_{1} \cup(X-F)\right\} \cap\left\{G_{2} \cup(X-F)\right\} .
$$

Since $G_{2}-F \in \mathscr{T}_{2} \cap \mathscr{P}(X-F) \subset \mathscr{T}_{1}$, we have $\left(G_{1} \cup G_{2}\right) \cap\left\{G_{1} \cup(X-F)\right\}$ $=G_{1} \cup\left(G_{2}-F\right) \in \mathscr{T}_{1}$. Moreover, $G_{2} \cup(X-F) \in \mathscr{T}_{1}$ since it is a cofinite subset. Hence, we have $G \in \mathscr{T}_{1}$, and similarly $G \in \mathscr{T}_{2}$. On the other hand, since $x \in G$ and $G \subset G_{1} \cup G_{2} \notin \mathscr{U}$, we have $G \notin \mathscr{T}(x, \mathscr{U})$. This contradicts that $\mathscr{T}(x, \mathscr{U}) \geqq \mathscr{T}_{1} \wedge \mathscr{T}_{2}$.

In the dual of $L_{T}(X)$, since $\theta$ is a congruence relation satisfying $\left(\Omega_{D}\right)$, $\theta$ satisfies $\left(\theta_{D}\right)$ by Lemma 2.1 . Hence, $\theta$ satisfies $\left(\theta_{D}\right)$ in $L_{T}(X)$ also. Therefore $L_{T}(X)$ is locally distributive.

REMARK. It follows from Theorem 1.1 that the above theorem is a generalization of [5, Theorems 3 and 4]. Moreover, Lemma 2.2 and Theorem 2.1 are lattice theoretical generalizations of [5, Lemma 9 and Theorem 5 (respectively)].

3. Standard elements in the dual of the lattice of $T_{1}$-topologies. Following [4], an element $a$ of a lattice $L$ is called standard when

$$
x \wedge(a \vee y)=(x \wedge a) \vee(x \wedge y) \text { for all } x, y \in L,
$$


and $a$ is called distributive when

$$
a \vee(x \wedge y)=(a \vee x) \wedge(a \vee y) \text { for all } x, y \in L .
$$

It follows from Theorems 1 and 3 of [4] that any standard element is distributive and that all standard elements form a sublattice of $L$.

Lemma 3.1. Let a be an element of an atomistic lattice $L$. The following three statements are equivalent.

$(\alpha)$ a is standard.

$(\beta)$ a is distributive.

$(\gamma)$ If $p$ is an atom of $L$ such that $p \leqq a \vee x$ and $p \leq x$ then $p \leqq a$.

Proof. It is easy to verify the implications $(\beta) \Rightarrow(\gamma) \Rightarrow(\alpha)$, and the details are omitted.

Let $X$ be an infinite set. We denote by $\mathscr{C}(X)$ the collection of all cofinite subsets of $X$. For any subset $A$ of $X$, it is evident that $\mathscr{S}(A)=\mathscr{P}(A) \cup \mathscr{C}(X)$ is a $T_{1}$-topology. Especially, $\mathscr{S}(X)$ is the discrete topology, $\mathscr{S}(\varnothing)$ is the cofinite topology and $\mathscr{S}(\{x\})$ is an atom of $L_{T}(X)$ for any $x \in X$. The set $\{\mathscr{S}(A) ; A \subset X\}$ forms a Boolean sublattice of $L_{T}(X)$, which coincides with the lattice $\Lambda_{0}$ appeared in [1].

THEOREM 3.1. Let $\mathscr{T}_{0}$ be an element of the lattice $L_{T}(X)$ of $T_{1}$-topologies on an infinite set $X$, and let $\mathscr{T}_{0} \neq \mathscr{S}(\varnothing)$. The following three statements are equivalent.

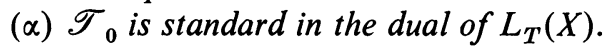

( $\beta$ ) $\mathscr{T}_{0}$ is distributive in the dual of $L_{T}(X)$.

$(\gamma) \mathscr{T}_{0}=\mathscr{S}(X-F)$ for some finite subset $F$ of $X$.

Proof. Since $L_{T}(X)$ is dual-atomistic, it follows from Lemma 3.1 that each of $(\alpha)$ and $(\beta)$ is equivalent to the following statement:

$(\delta)$ If $\mathscr{T}(x, \mathscr{U}) \geqq \mathscr{T}_{0} \wedge \mathscr{T}$ and $\mathscr{T}(x, \mathscr{U}) \geq \mathscr{T}$ then $\mathscr{T}(x, \mathscr{U}) \geqq \mathscr{T}_{0}$.

First, we shall prove that $(\gamma)$ implies $(\delta)$. Let $\mathscr{T}(x, \mathscr{U}) \geqq \mathscr{S}(X-F) \wedge \mathscr{T}$. If $\mathscr{T}(x, \mathscr{U}) \geq \mathscr{T}$, then there exists $G \in \mathscr{T}$ with $G \notin \mathscr{T}(x, \mathscr{U})$, whence $x \in G \notin \mathscr{U}$. Since $G-F \in \mathscr{S}(X-F) \cap \mathscr{T} \subset \mathscr{T}(x, \mathscr{U})$ and $G-F \notin \mathscr{U}$, we have $G-F \subset X-\{x\}$, whence $x \in F$. Hence, $\mathscr{S}(X-F) \leqq \mathscr{T}(x, \mathscr{U})$. Therefore, $\mathscr{T}_{0}=\mathscr{S}(X-F)$ satisfies $(\delta)$.

Next, we assume that $\mathscr{T}_{0}$ satisfies $(\delta)$, and we put $F=\left\{x \in X ;\{x\} \notin \mathscr{T}_{0}\right\}$. We shall prove that $\mathscr{T}_{0}=\mathscr{S}(X-F)$. Since $\{x\} \in \mathscr{T}_{0}$ for every $x \in X-F$, we have $\mathscr{P}(X-F) \subset \mathscr{T}_{0}$, whence $\mathscr{S}(X-F) \leqq \mathscr{T}_{0}$. If $\mathscr{S}(X-F)<\mathscr{T}_{0}$, then there would exist $G \in \mathscr{T}_{0}$ such that $G \notin \mathscr{S}(X-F)$. Then we have $G \cap F \neq \varnothing$. We take $x \in G \cap F$ and put $\mathscr{T}=\mathscr{S}(\{x\})$. Since $G \notin \mathscr{C}(X)$, the set $A=X-G$ is infinite. Hence, there exists a nonprincipal ultrafilter $\mathscr{U}$ on $X$ such that $A \cup\{x\} \in \mathscr{U}$. Since $\{x\} \notin \mathscr{T}_{0}$, we have $\mathscr{T}_{0} \wedge \mathscr{T}=\mathscr{S}(\varnothing) \leqq \mathscr{T}(x, \mathscr{U})$. Moreover, $\mathscr{T} \mathscr{T}(x, \mathscr{U})$ since $\{x\} \in \mathscr{T}$. On the other hand, we have 
$(A \cup\{x\}) \cap G=\{x\} \notin \mathscr{U}$ since $\mathscr{U}$ is nonprincipal. Since $A \cup\{x\} \in \mathscr{U}$, we have $G \notin \mathscr{U}$. Hence, $G \notin \mathscr{T}(x, \mathscr{U})$, and therefore $\mathscr{T}_{0} \mathscr{T}(x, \mathscr{U})$. This contradicts our assumption. Thus we get $\mathscr{T}_{0}=\mathscr{S}(X-F)$.

We shall prove that $F$ is finite. Since $\mathscr{T}_{0} \neq \mathscr{S}(\varnothing)$, there exists $x \in X-F$. If $F$ were infinite, then there would exist a subset $A$ of $F$ such that both $A$ and $X-A$ are infinite. Put $\mathscr{T}=\mathscr{S}(\varnothing) \cup\{G \cap(A \cup\{x\}) ; G \in \mathscr{C}(X)\}$. It is evident that $\mathscr{T} \in L_{T}(X)$. Since $X-(A \cup\{x\})$ is infinite, there exists a nonprincipal ultrafilter $\mathscr{U}$ which contains this set. We have $\mathscr{T}_{0} \mathscr{T}(x, \mathscr{U})$ since $\{x\} \in \mathscr{T}_{0}$. Since $A \cup\{x\} \notin \mathscr{U}$, we have $A \cup\{x\} \notin \mathscr{T}(x, \mathscr{U})$. Hence, $\mathscr{T} \neq \mathscr{T}(x, \mathscr{U})$. If $G \in \mathscr{C}(X)$, then $G \cap(A \cup\{x\}) \cap F \supset G \cap A \neq \varnothing$, since $A$ is infinite. Hence, $G \cap(A \cup\{x\}) \notin X-F$, whence $G \cap(A \cup\{x\}) \notin \mathscr{S}(X-F)=$ $\mathscr{T}_{0}$. Therefore, $\mathscr{T}_{0} \wedge \mathscr{T}=\mathscr{S}(\varnothing) \leqq \mathscr{T}(x, \mathscr{U})$. This contradicts our assumption. Thus, it has been proved that $(\delta)$ implies $(\gamma)$. This completes the proof.

It is shown in [2, Chapter III, $\S 9$ ] that an element of a lattice $L$ is neutral if and only if it is standard in both $L$ and its dual and that if a neutral element has a complement then it is also neutral. Hence, it follows from the above theorem that

COROLlaRY. The lattice $L_{T}(X)$ has no neutral element except the greatest element $\mathscr{S}(X)$ and the least element $\mathscr{S}(\varnothing)$.

Finally, we remark that the congruence relation $\mathscr{T}_{1} \equiv \mathscr{T}_{2}(\theta)$ in $L_{T}(X)$ defined in Theorem 2.2 coincides with the relation defined by each of the following equations:

$$
\mathscr{T}_{1} \wedge \mathscr{S}(X-F)=\mathscr{T}_{2} \wedge \mathscr{S}(X-F), \quad \mathscr{T}_{1} \vee \mathscr{S}(F)=\mathscr{T}_{2} \vee \mathscr{S}(F)
$$

\section{REFERENCES}

1. R. W. Bagley, On the characterization of the lattice of topologies, J. London Math. Soc. 30 (1955), 247-249. MR 16, 788.

2. G. Birkhoff, Lattice theory, 3rd ed., Amer. Math. Soc. Colloq. Publ., vol. 25, Amer. Math. Soc., Providence, R.I., 1967. MR 37 \#2638.

3. O. Fröhlich, Das Halbordnungssystem der topologischen Räume auf einer Menge, Math. Ann. 156 (1964), 79-95. MR 29 \#4023.

4. G. Grätzer and E. T. Schmidt, Standard ideals in lattices, Acta Math. Acad. Sci. Hungar. 12 (1961), 17-86. MR 24 \#A3099.

5. R. E. Larson and W. J. Thron, Covering relations in the lattice of $T_{1}$-topologies, Trans. Amer. Math. Soc. 168 (1972), 101-111. MR 45 \#5942.

6. F. Maeda and S. Maeda, Theory of symmetric lattices, Die Grundlehren der math. Wissenschaften, Band 173, Springer-Verlag, New York and Berlin, 1970. MR 44 \#123.

7. A. K. Steiner, The lattice of topologies: structure and complementation, Trans. Amer. Math. Soc. 122 (1966), 379-398. MR 32 \#8303.

Department of Mathematics, Ehime University, Bunkyo-Cho, Matsuyama-Shi, JAPAN 